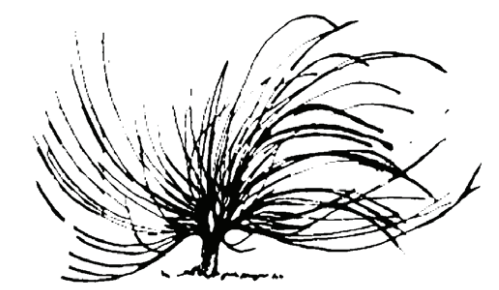

\title{
La investigación como una oportunidad para valorar la práctica profesional docente
}

\author{
Magdalena Alfaro Rodríguez ${ }^{1}$ \\ Marlene Aguirre Chaves ${ }^{2}$ \\ Universidad Nacional, Costa Rica \\ Heredia, Costa Rica
}

\begin{abstract}
Resumen
El análisis de la práctica pedagógica debe ser una actividad continua y sistemática, en procura del mejoramiento de los procesos de enseñanza y aprendizaje. La investigación de dichas prácticas es una excelente forma de analizar estos procesos. A través de ella, los(as) docentes llevan a cabo una introspección de todo lo que hacen, de cómo se sienten y cómo están conceptualizando su labor. Todo esto a través de una reflexión sobre y en la acción en la que se pueden visualizar los aciertos y desaciertos de su trabajo; así como todo lo que se debe mejorar. En este artículo se detallan algunas técnicas como una forma de acercamiento a la investigación de aula y de investigación acción, como formas de valorar la labor docente en el contexto de práctica "dirigida" como es el caso de la que se lleva a cabo en la División de Educología, de las prácticas en las carreras en la enseñanza, con excepción de la enseñanza del inglés.
\end{abstract}

Recibido: 30 de octubre de 2011 - Aprobado: 30 de mayo de 2012

1 Máster en Educación con énfasis en Docencia Universitaria y Licenciada en Música con énfasis en Educación Musical. Actualmente es docente de la División de Educología (CIDE) de la Universidad Nacional y docente del Ministerio de Educación Pública.

2 Licenciada en Ciencias de la Educación de la Universidad Nacional. Académica en la División de Educología de del Centro de Investigación y Docencia en Educación de la Universidad Nacional, Costa Rica. 
Palabras clave: práctica docente, investigación de aula, investigación-acción, técnicas de recogida de datos.

\begin{abstract}
Reflecting on the pedagogical practice must be a continuous and systematic activity in search of the teaching and learning process improvement. The research on that field represents an excellent way of analyzing the process through a teacher's introspection on his own feelings and opinions. This reflection allows the teacher to recognize his strengths and weaknesses in order to improve his teaching practice. This paper presents some techniques on how to approach the classroom research and the action-research as methods for valuing the guided teaching practicum of most of the practices in careers in teaching, except for the teaching of English of Educology Division.
\end{abstract}

Keywords: Student-teaching practicum, classroom research, action-research, data collection techniques.

\title{
Introducción
}

$\mathrm{E}$ nfrentarse a la práctica docente es uno de los momentos más importantes en el proceso de formación de todos(as) los estudiantes de las carreras de educación. Dentro de este proceso, se debe analizar la forma de valorar dichas prácticas; tema que está en discusión en todas las cátedras y grupos de docentes que deben enfrentar cursos de práctica docente ya que resulta bastante complejo valorar esas experiencias, únicas para cada uno(a) de los(as) practicantes y en los que confluyen factores de diferentes índoles; sobre todo cuando se trata de cursos como el que se imparte en la División de Educología, del Centro de Investigación y Docencia en Educación (CIDE), que pasó de ser una práctica supervisada, en la que el docente del curso supervisaba el proceso vivido por cada estudiante en el proceso de práctica, a ser una práctica dirigida, en la que el estudiante es acompañado y valorado por los docentes con el que realizan dicha práctica; trayendo todo esto una serie de inconvenientes y cambios en la forma de concebir y llevar a cabo este proceso. 


\section{Valoración del proceso}

En el contexto de la práctica dirigida debe analizarse, desde varias aristas; la del docente a cargo de la cátedra o del Seminario, la del docente colaborador o co-formador y la del (la) propio(a) alumno(a) que es el que está viviendo el proceso.

Es necesaria una revisión exhaustiva del proceso de aula, de lo que pasa con los estudiantes, cómo se siente el (la) docente, cómo los elementos del currículo interactúan en la complejidad de un proceso educativo específico. Al respecto anota Shulman, L. (citado por Wittrok, 1989: 9): "Los que investigan la enseñanza están comprometidos en la tarea de comprender sus fenómenos, de aprender cómo mejorar su realización, de descubrir mejores maneras de preparar a los individuos que quieren enseñar". Que mejor que tener una comprensión de estos procesos desde el proceso mismo, desde lo que ocurre en las interacciones entre alumnos y profesor y entre alumnos y alumnos. Recoger sus inquietudes, sus deseos, sus emociones, sus sentimientos y en medio de esto sus aprendizajes. Esto obedece a procesos holísticos y cualitativos más que brindar una nota al final del curso.

Para evitar que estos procesos de evaluación caigan sobre una evaluación de corte cuantitativo, en la que el evaluar se convierte en una rendición de cuentas de ese proceso, es necesario recoger la mayor cantidad de información que pueda redundar en procesos más cualitativos que sin lugar a dudas son los más beneficiosos.

Según Shulman L. (citado por Wittrok, 1989:12) surgen algunas preguntas, sobre cómo abordar estos procesos, a saber: “¿Cómo debería estudiarse la enseñanza?, ¿Por dónde se empieza?” Existe una gran diversidad de respuestas dependiendo de la postura epistemológica en la cual se encuentre y el encuadre del problema por resolver. Es importante adoptar un tipo de investigación, según los intereses y los problemas que se deseen abordar, mediante el paradigma positivista si se quiere cuantificar datos o el naturalista si lo que se quiere es interpretar hechos y fenómenos.

\section{La investigación de aula como forma de abordar el análisis del pro- ceso de práctica docente}

La investigación cualitativa es uno de los enfoques que pueden ser muy valiosos, según Wood(1987 citado por Sandin, 2003: 154), este 
tipo de estudio hace una "descripción de un modo de vida, de una raza o un grupo de individuos" y específicamente en investigación de aula, con estudios etnográficos, en los cuales "el objeto de la etnografía educativa es aportar valiosos datos descriptivos de los contextos, actividades y creencias de los participantes en los escenarios educativos", Goetz y LeCompte (1988 citado por Sandin, 2003: 155).

Todos los profesionales reflexionan sobre lo que hacen, no sólo al terminar su labor sino también durante el proceso. Se enfrentan a un proceso de análisis en el que tratan de buscar soluciones a los problemas enfrentados y esto se da en una forma natural, lo cual es denominado por Shön (1998) como la reflexión desde la acción o el conocer desde la acción. Sin embargo, no debe quedarse ahí sino que es necesaria una reflexión desde y sobre su práctica ya que esto le permitirá descentrarse de las rutinas y de los conocimientos tácitos que tenga formados de sus anteriores experiencias. Puede reflexionar sobre sus incertidumbres y la de sus alumnos, acerca de aquellas formas de enseñar que le proporcionen estabilidad y el control de las situaciones, sin percatarse de que es necesario que también enfrenten la dificultad y lo complejo. Debemos aprender a vivir con la incertidumbre y no como nos han querido enseñar a vivir desde hace milenios, a hacer cualquier cosa para evitar la incertidumbre" (Morín, 2005: 440), esta situación se da porque en las relaciones dentro de la ecología del aula nunca estaremos seguros de que es lo mejor, las interpretaciones y la construcción del conocimiento son procesos sociales pero a la vez individuales y obedece a una realidad cambiante y compleja.

En el marco de prácticas pedagógicas complejas, resulta una condición sine cua non el mejoramiento pedagógico a través de la reflexión y el análisis; lo cual debe constituirse en una actividad propia del quehacer docente y dentro de este proceso, la investigación cualitativa brinda excelentes aportes ya que permite, entre otras cosas, analizar procesos humanos desde una perspectiva profunda y específica del grupo de personas que participan en esos procesos, así como detectar algunos elementos que en la investigación tradicional son difíciles de determinar. Este tipo de estudio se lleva a cabo desde dentro, desde el proceso mismo. Para analizar los procesos educativos se debe dar un acercamiento hacia las personas, hacia los grupos, de modo que se reviertan esos procesos investigativos en el mejoramiento de la práctica educativa. 
¿Cómo abordar la práctica profesional docente a través de la investigación cualitativa? Para empezar es necesario decir que se debe de conceptualizar al docente como un investigador, no sólo como un dador de clases. A lo que Stenhouse (1998) anota que,

La valiosa actividad desarrollada por el profesor y los estudiantes tienen en sí niveles y criterios inmanentes y la tarea de apreciación consiste en perfeccionar la capacidad, por parte de los estudiantes, para trabajar según dichos criterios, mediante una relación crítica respecto al trabajo realizado. En este sentido, la evaluación viene a ser la enseñanza de la autoevaluación (p.139).

Dentro de un planteamiento de valoración cualitativa, de los procesos en su propia práctica, se deben conocer las observaciones, las reflexiones, las valoraciones que haga el alumno sobre el proceso, sobre el currículo y también sobre el docente.

La evaluación en educación es una actividad científica, cuantificable pero también cualificable, que cubre una gran cantidad de aspectos y que solamente nos hemos quedado en la calificación del aprendizaje, en la medición de simples contenidos como si éstos estuvieran desligados de todos esos elementos que se escapan de la racionalidad y por tanto de lo medible.

Se debe aplicar una evaluación holística, vista como parte del proceso, en la que ésta se convierte en una tarea compleja que aporta elementos de interpretación de la docencia como situación dinámica susceptible de constante mejora y modificación, dentro de un concepto integral, con la participación activa de todos y todas las involucradas en este proceso.

Para evaluar hay que comprender. Cabe afirmar que las evaluaciones convencionales de tipo objetivo no van destinadas a comprender el proceso educativo. La adopción de un enfoque holístico implica admitir que ciertos datos (como los resultados obtenidos por los alumnos) son potencialmente importantes en determinados contextos. (Stenhouse, 1998, pp. 156-157). 


\section{El aporte de la Investigación Acción para valorar el proceso de práctica docente}

La investigación acción en los últimos años, ha hecho una importante contribución al campo de la educación, ya que entre otros aspectos, permite llevar a cabo una práctica reflexiva, conceptualizando al docente como un investigador de su propia práctica ya que por mucho tiempo se concibió como algo separado la labor docente de la labor investigativa. Así, "los datos se recogen en el transcurrir de la práctica en el aula, se analizan e interpretan y vuelven a generar nuevas preguntas e hipótesis para ser sometidas a indagación" (Latorre, 2004, p. 10).

El proceso de investigación es cíclico, aspecto que se puede demostrar en el siguiente gráfico circular.

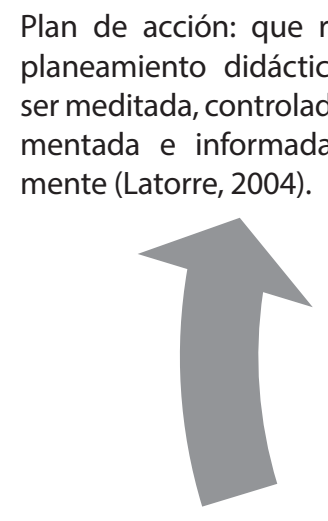

Reflexión sobre y en la acción: la investigación supone una conversación con la situación problemática o el fenómeno en análisis (Latorre, 2004).

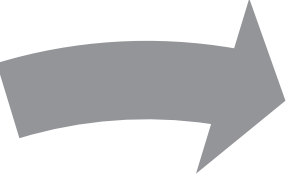

Acción: puesta en práctica del plan de acción o planeamiento didáctico.
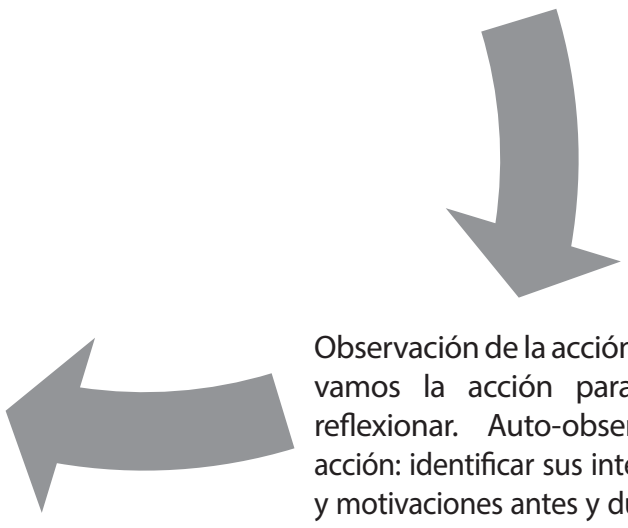

Observación de la acción: Observamos la acción para poder reflexionar. Auto-observar su acción: identificar sus intenciones y motivaciones antes y durante el proceso (Latorre, 2004).

Este proceso cíclico en un inicio podría parecer complejo, pero los(as) y las docentes no están acostumbrados a sistematizar sus experiencias y a reflexionar sobre ellas, y si lo hacen en muchas ocasiones no pasan de un análisis general. Con el tiempo se puede constituir en una cultura investigativa y se lleva a cabo de manera más "mecánica" en relación con el procedimiento. Entonces, redundará en una transformación de la labor docente; empezando con el educador, quien debe construir una cultura investigativa de su labor como docente y en la transformación de las prácticas docentes. 
Algunas técnicas que se pueden utilizar en la investigación de aula y en la investigación-acción en la recogida de datos podrían ser los(as) siguientes:

- La observación participante: en esta técnica se puede utilizar el diario de campo para registrar todo lo observado al inicio, durante y al final del proceso. En este diario se pueden registrar diversos tipos de observaciones, interpretaciones, dibujos, vídeos, fotografías, poesía, trabajos grupales, entre otros. El (la) docente deberá registrar hasta los elementos más insignificantes y desarrollar y agudizar todos los sentidos de modo que pueda luego de cada una de las clases interpretar y repensar su práctica. Debe desarrollar la observación como una técnica enriquecedora en este tipo de estudio; así como también el uso de los otros sentidos: el olfato, el tacto, el gusto y el oído. En cuanto a la observación, deberá ser desarrollada con agudeza, debe ser una observación continua, sistemática e intencionada.

- $\quad$ Notas de campo. "Son registros que contienen información registrada en vivo por el investigador y que contienen descripciones y reflexiones percibidas en el contexto natural" (Latorre, 2004, p. 58).

- Diario del investigador: Esta técnica "recoge observaciones, reflexiones, interpretaciones, hipótesis y explicaciones de lo que ha ocurrido" (Latorre, 2004, p.60).

- Memorandos. Los memorandos son notas personales-conceptuales escritas por el investigador con la finalidad de analizar información. El análisis suele ser bastante breve y pueden cruzarse referencias útiles con otros datos de campo, diarios, etc." (Latorre, 2004, p. 63).

- $\quad$ Registros anecdóticos. Esta técnica permite recoger lo que se ha percibido con los sentidos, por ejemplo, con el sentido auditivo, se debe escuchar más que oír. Poner mucha atención a lo que dicen sus alumnos(as), a lo que escuchan. Puede hacerles preguntas sobre sus niveles de percepción, sobre lo que les gusta escuchar y por qué, pedirles que lean o escuchen poesía y escriban reacciones o canciones, por ejemplo, para interpretar, luego, el sentido de lo que hagan. El tacto, el olfato y el gusto también deben ser desarrollados tanto por el(la) docente investigador(a), como por los(as) alumnos(as) para interpretar los procesos de enseñanza y de aprendizaje, sobre cómo se están percibiendo y sobre cómo están comprendiendo los contenidos y las actividades o experiencias de 
aula. Se pueden llevar a cabo diversas actividades (de acuerdo con lo investigado), tales como probar diversos sabores, oler diferentes tipos de objetos y productos como perfumes, flores, con olores agradables o desagradables y palpar diferentes tipos de texturas. Es conveniente llevar a cabo actividades en las que se tenga algún tipo de contacto físico, como tomarse de las manos, tocarse el hombro, hacer espejo con movimientos corporales, abrazarse, entre otras; por supuesto que dentro del marco del respeto a la integridad física y emocional de los(as) demás. Este tipo de actividades permitirá conocer más profundamente a los(as) alumnos(as), en relación con sus gustos, intereses y estilos de aprendizaje (visual, auditivo, entre otros), además de algunos rasgos de su personalidad.

- Diversas técnicas grupales. La aplicación y sistematización deben mostrar diversas dimensiones de la acción investigativa, entre ellas se pueden enumerar las siguientes: el dibujo, la poesía, la dramatización, la canción, los socio-dramas, los grupos de discusión, entre otras.

- $\quad$ El portafolio. Éste constituye un reflejo de lo que el estudiante ha construido durante todo el proceso de aprendizaje, favorece la metacognición y la reflexión acerca de los procesos vividos; es una técnica interesante, es como un retrato del alumno, de cómo se ha acercado al conocimiento y al aprendizaje.

- $\quad$ Entrevistas en profundidad. Según Taylor y Bodgan (1987, p.101) son reiterados encuentros cara a cara entre entrevistador y entrevistados, dirigidos hacia la comprensión de las perspectivas que tienen los informantes respecto de sus vidas, experiencias o situaciones, tal como las expresan con sus propias palabras”. Esta técnica se puede utilizar cuantas veces sea necesario, sobre todo para profundizar en algunos aspectos que mediante la observación no quedan del todo claros y para llevar a cabo el estudio de algún(os) caso(s), como insumo de la investigación de aula.

- $\quad$ Escalas de medida. Según Latorre, (2004, p. 65), "las escalas de medida se utilizan para determinar las diferencias de grado o intensidad entre los individuos respecto a algún objeto actitudinal".

- Grabaciones en vídeo: Se pueden registrar con este instrumento diferentes momentos de la clase que nos permitan reflexionar sobre lo acontecido en un período específico. 
- Las técnicas grupales:

- Son un conjunto de procedimientos que se utilizan para lograr con eficiencia las metas propuestas. La técnica es el diseño, el modelo congruente y unitario que se forma con base en diferentes modos, a partir de los cuales se pretende que un grupo funcione, sea productivo y alcance otras metas más(Barrantes, 2002, p. 217).

Estas técnicas, además de formar parte de la didáctica, también es una oportunidad para recabar información importante de los informantes, sobre todo en los aspectos sociales, de relación interpersonal y de negociación de significados. Se pueden llevar a cabo juegos, discusión en grupos, el diálogo simultáneo, mesas redondas, grupos de confrontación, sociodramas, entre otras.

Podrían enumerarse otras muchas, pero es necesario que del docente surjan esas ideas propias de cada caso en particular, ya que cada uno de ellos posee características muy particulares y el abordaje debe ser por ende pertinente a dichos procesos.

En cuanto a la metodología en la investigación cualitativa se utiliza el método inductivo, es decir, analizar los hechos a partir de los elementos particulares para así establecer algunas interpretaciones de modo general.

Es importante anotar que el docente o la docente debe llevar a cabo un análisis no sólo de la información recopilada, sino también de la realimentación que se da en el proceso mediante la interacción con los alumnos, cuando se lleva a cabo la clase, que haga reflexiones sobre los trabajos realizados, sobre las técnicas utilizadas, sobre cómo se sintieron, sobre lo que aprendieron; lo cual redundará en el desarrollo de procesos metacognitivos y en material para definir algunos patrones de comportamiento, que sirvan de insumo para la construcción del informe final del proceso investigativo.

La información recopilada se debe triangular con las evaluaciones, percepciones y valoraciones hechas por los profesores colaboradores y las del(la) docente encargado(a) del curso.

Todo este análisis deberá ser devuelto a los estudiantes con los que se trabajó y por supuesto al coformador(a) y al profesor de la cátedra o encargado del curso de práctica docente; de modo que sirva como una autoevaluación, una evaluación de los estudiantes y un insumo para el profesor del curso para evaluar de una manera más real y profunda el proceso vivido durante la práctica docente. 
Esto constituye el trabajo final de investigación ya que se llevaría a cabo en dos ciclos lectivos: el primero con la práctica docente y la recolección de la información y el segundo con la sistematización de dicha información, culminando con un informe presentado en la feria de investigación.

\section{Consideraciones finales}

Las prácticas docentes son complejas y se hace necesario un análisis profundo de las ellas y, que mejor que a través de la investigación-acción, pues ésta le permitirá entre otras cosas valorar dicha práctica a la luz de las reflexiones y análisis de lo que pasa en el 'aula'. Asimismo, el practicante podrá mejorar y reorientar su labor docente más allá de una supervisión o valoración externa, ya sea por parte de un docente universitario o de un profesor colaborador.

Estos procesos promueven en el estudiante practicante una actitud metacognitiva y metadidácta, pues se produce una reflexión profunda de su quehacer, formándose a su vez como un investigador y no sólo como un dador de clases. Esto cambiaría profundamente la concepción de docente que hasta la fecha en muchos de los espacios se ha tenido.

\section{Referencias bibliográficas}

Barrantes, R. (1999). Investigación. Un camino al conocimiento. Un enfoque cuantitativo y cualitativo. San José, Costa Rica: EUNED.

Latorre, A. (2004). La investigación-acción. Conocer y cambiar la práctica educativa. Barcelona: Graó.

Morín, E. (2005). Introducción al pensamiento complejo. Barcelona, España: Gedisa.

Sandin, E. (2003). Investigación cualitativa en educación. Fundamentos y tradiciones. Madrid, España: Mc Graw-Hill.

Shön, D. El profesional reflexivo. Cómo piensan los profesionales cuando actúan. Barcelona, España: Paidós.

Stenhouse, L. (1998). Investigación y desarrollo del currículum. Madrid, España: Morata.

Taylor, S. J. y Bodgan, R. (1987). Introducción a los métodos cualitativos de investigación. Barcelona, España: Paidós.

Wittrock. M. (1989). La investigación de la enseñanza. Barcelona, España: Paidós. 